\title{
The mod 2 dual Steenrod algebra as a subalgebra of the mod 2 dual Leibniz-Hopf algebra
}

\author{
Neşet Deniz Turgay ${ }^{1}$ • Shizuo Kaji ${ }^{2}$
}

Received: 18 November 2015 / Accepted: 23 October 2016 / Published online: 15 November 2016

(C) Tbilisi Centre for Mathematical Sciences 2016

\begin{abstract}
The mod 2 Steenrod algebra $\mathcal{A}_{2}$ can be defined as the quotient of the mod 2 Leibniz-Hopf algebra $\mathcal{F}_{2}$ by the Adem relations. Dually, the mod 2 dual Steenrod algebra $\mathcal{A}_{2}^{*}$ can be thought of as a sub-Hopf algebra of the mod 2 dual Leibniz-Hopf algebra $\mathcal{F}_{2}^{*}$. We study $\mathcal{A}_{2}^{*}$ and $\mathcal{F}_{2}^{*}$ from this viewpoint and give generalisations of some classical results in the literature.
\end{abstract}

Keywords Leibniz-Hopf algebra $\cdot$ Steenrod algebra $\cdot$ Adem relation · Hopf algebra Conjugation · Antipode

Mathematics Subject Classification 55S10 $\cdot 16 \mathrm{~T} 05 \cdot 57 \mathrm{~T} 05$

Communicated by Stewart Priddy.

The second named author was partially supported by KAKENHI, Grant-in-Aid for Young Scientists (B) 26800043 and JSPS Postdoctoral Fellowships for Research Abroad.

Shizuo Kaji

skaji@yamaguchi-u.ac.jp

Neşet Deniz Turgay

Deniz_Turgay@Yahoo.com

1 Department of Mathematics, Faculty of Arts and Sciences, Eastern Mediterranean University, Gazimagusa, TRNC, Mersin 10, Turkey

2 Department of Mathematical Sciences, Faculty of Science, Yamaguchi University, 1677-1, Yoshida, Yamaguchi 753-8512, Japan 


\section{The mod 2 Leibniz-Hopf algebra and its dual}

Let $\mathcal{F}_{2}$ be the free associative algebra over $\mathbb{F}_{2}$ generated by the indeterminates $S^{1}, S^{2}, S^{3}, \ldots$ of degree $\left|S^{i}\right|=i$. We often denote the unit 1 by $S^{0}$. This algebra is equipped with a co-commutative co-product given by

$$
\Delta\left(S^{n}\right)=\sum_{i=0}^{n} S^{i} \otimes S^{n-i}
$$

which makes it a graded connected Hopf algebra. This algebra $\mathcal{F}_{2}$ is often called the mod 2 Leibniz-Hopf algebra. As an $\mathbb{F}_{2}$-module, $\mathcal{F}_{2}$ has the following canonical basis:

$$
\left\{S^{I}:=S^{i_{1}} S^{i_{2}} \ldots S^{i_{n}} \mid I=\left(i_{1}, i_{2}, \ldots, i_{n}\right) \in \mathbb{N}^{n}, 0 \leq n<\infty\right\}
$$

where we regard $S^{I}=1$ when $n=0$.

Note that the integral counterpart of $\mathcal{F}_{2}$ is called the Leibniz-Hopf algebra and is isomorphic to the ring of non-commutative symmetric functions [7] and the Solomon Descent algebra [17]. Its graded dual is the ring of quasi-symmetric functions with the outer co-product, which has been studied by Hazewinkel, Malvenuto, and Reutenauer in $[8-12]$.

The mod 2 Steenrod algebra $\mathcal{A}_{2}$ is defined to be the quotient Hopf algebra of $\mathcal{F}_{2}$ by the ideal generated by the Adem relations:

$$
S^{i} S^{j}-\sum_{k=0}^{\lfloor i / 2\rfloor}\left(\begin{array}{c}
j-k-1 \\
i-2 k
\end{array}\right) S^{i+j-k} S^{k}
$$

Denote the quotient map by $\pi: \mathcal{F}_{2} \rightarrow \mathcal{A}_{2}$ and $S q^{i}=\pi\left(S^{i}\right)$. It is well-known (see, for example, [18]) that the admissible monomials

$$
\left\{S q^{J}:=S q^{j_{1}} S q^{j_{2}} \cdots S q^{j_{n}} \mid J=\left(j_{1}, j_{2}, \ldots, j_{n}\right) \in \mathbb{N}_{>0}^{n}, 0 \leq n<\infty, j_{k-1} \geq 2 j_{k} \forall k\right\}
$$

form a module basis for $\mathcal{A}_{2}$. We will adhere to this purely algebraic definition and will not use any other known facts about $\mathcal{A}_{2}$.

By taking the graded dual of $\pi$, we obtain the following inclusion of Hopf algebras

$$
\pi^{*}: \mathcal{A}_{2}^{*} \rightarrow \mathcal{F}_{2}^{*}
$$

$\mathcal{F}_{2}^{*}$ is given a module basis $S_{I}$ dual to $S^{I}$, that is,

$$
\left\langle S^{I^{\prime}}, S_{I}\right\rangle=\left\{\begin{array}{ll}
1 & \left(I=I^{\prime}\right) \\
0 & \left(I \neq I^{\prime}\right)
\end{array} .\right.
$$


Similarly, we have the dual basis $\left\{S q_{J} \mid J\right.$ admissible $\}$ for $\mathcal{A}_{2}^{*}$ determined by

$$
\left\langle S q^{J^{\prime}}, S q_{J}\right\rangle=\left\{\begin{array}{ll}
1 & \left(J=J^{\prime}\right) \\
0 & \left(J \neq J^{\prime}\right)
\end{array} .\right.
$$

The commutative product among the basis elements in $\mathcal{F}_{2}^{*}$ is given by the overlapping shuffle product (see $\$ 2$ ) and the co-product is given by

$$
\Delta\left(S_{a_{1}, \ldots, a_{n}}\right)=S_{a_{1}, \ldots, a_{n}} \otimes 1+1 \otimes S_{a_{1}, \ldots, a_{n}}+\sum_{i=1}^{n-1} S_{a_{1}, \ldots, a_{i}} \otimes S_{a_{i+1}, \ldots, a_{n}}
$$

The purpose of this paper is to deduce some of the classical results on $\mathcal{A}_{2}^{*}$ and its generalisations by considering it as a subalgebra of $\mathcal{F}_{2}^{*}$. We are particularly interested in the following problems.

Problem 1 (i) Determine the coefficients in

$$
\pi^{*}\left(S q_{J}\right)=\sum_{I} C_{J}^{I} S_{I}
$$

for all admissible sequences $J$. This is important since in the dual it is equivalent to computing the coefficients of the Adem relations

$$
S q^{I}=\sum_{J: \text { admissible }} C_{J}^{I} S q^{J}
$$

for all sequences $I$.

(ii) Give an expansion of the dual Milnor bases in terms of the dual admissible monomial bases, i.e., determine the coefficient $B_{J}^{L}$ in

$$
\xi^{L}=\sum_{J: \text { admissible }} B_{J}^{L} S q_{J}
$$

where $\xi_{n}=S q_{2^{n-1}, 2^{n-2} \ldots 2^{1}, 2^{0}}$ and $\xi^{L}=\xi_{1}^{l_{1}} \xi_{2}^{l_{2}} \ldots \xi_{n}^{l_{n}}$ for $L=\left(l_{1}, l_{2}, \ldots, l_{n}\right)$.

(iii) Generalise Milnor's conjugation formula [13] in $\mathcal{A}_{2}^{*}$ to $\mathcal{F}_{2}^{*}$. The formula for $\mathcal{A}_{2}^{*}$ is:

$$
\chi\left(\xi_{n}\right)=\sum_{\alpha} \prod_{i=1}^{l(\alpha)} \xi_{\alpha(i)}^{2^{\sigma(i)}}
$$

where $\alpha=(\alpha(1)|\alpha(2)| \ldots \mid \alpha(l(\alpha))$ runs through all the compositions of the integer $n$ and $\sigma(i)=\sum_{j=1}^{i-1} \alpha(j)$. 
Several different methods are known for resolving (i) and (ii) (see for example, $[15,19]$ ), but our argument (Sect. 4 ) is new in that it is purely combinatorial using the overlapping shuffle product on $\mathcal{F}_{2}^{*}$. We implemented our algorithm into a Maple code [16]. In Sect. 5 we discuss the conjugation (or antipode) in $\mathcal{F}_{2}^{*}$ and give an answer to (iii). Finally, we give an explicit duality between the conjugation invariants in $\mathcal{F}_{2}$ and $\mathcal{F}_{2}^{*}$ in Sect. 6.

\section{Overlapping Shuffle product}

We recall the definition of the overlapping shuffle product ([2, Section 2],[8]). Let $\mathcal{W}$ be the set of finite sequences of natural numbers:

$$
\mathcal{W}=\left\{\left(i_{1}, i_{2}, \ldots, i_{n}\right) \mid 0 \leq n<\infty\right\} .
$$

Note that we allow the length 0 sequence. Consider the $\mathbb{F}_{2}$-module $\mathbb{F}_{2}\langle\mathcal{W}\rangle$ freely generated by $\mathcal{W}$. For a sequence $I=\left(i_{1}, i_{2}, \ldots, i_{n}\right)$, denote its tail partial sequence $\left(i_{k}, i_{k+1}, \ldots, i_{n}\right)$ by $I_{k}$. When $n<k$, we regard $I_{k}$ as the length 0 sequence. We use the convention

$$
\begin{aligned}
& \left(a_{1}, a_{2}, \ldots, a_{k},\left(b_{1}, \ldots, b_{i}\right)+\left(c_{1}, \ldots, c_{j}\right)\right) \\
& \quad:=\left(a_{1}, a_{2}, \ldots, a_{k}, b_{1}, \ldots, b_{i}\right)+\left(a_{1}, a_{2}, \ldots, a_{k}, c_{1}, \ldots, c_{j}\right) .
\end{aligned}
$$

The overlapping shuffle product on $\mathbb{F}_{2}\langle\mathcal{W}\rangle$ is defined as follows:

Definition 1 For $A=\left(a_{1}, a_{2}, \ldots, a_{n}\right)$ and $B=\left(b_{1}, b_{2}, \ldots, b_{m}\right)$, define their product inductively by

$$
A \cdot B:= \begin{cases}A & (m=0) \\ B & (n=0) \\ \sum_{0 \leq i \leq n}\left(a_{1}, \ldots, a_{i}, b_{1}, A_{i+1} \cdot B_{2}\right) & \\ +\sum_{1 \leq i \leq n}\left(a_{1}, \ldots, a_{i}+b_{1}, A_{i+1} \cdot B_{2}\right) & \text { (otherwise) } .\end{cases}
$$

The product on $\mathbb{F}_{2}\langle\mathcal{W}\rangle$ is defined by the linear extension of the above.

We say a term in $A \cdot B$ is $a$-first if there exists $k$ such that $a_{k}$ goes ${ }^{1}$ to an entry to the left of $b_{k}$ and $a_{i}$ goes to the same entry as $b_{i}$ (that is, the entry makes $a_{i}+b_{i}$ ) for all $i<k$. For example, $\left(a_{1}+b_{1}, a_{2}, b_{2}, b_{3}, a_{3}\right)$ is $a$-first while $\left(a_{1}+b_{1}, b_{2}, a_{2}, a_{3}, b_{3}\right)$ is not. Observe that

Lemma 1 For equal length sequences, we have

$$
\left(a_{1}, \ldots, a_{n}\right) \cdot\left(b_{1}, \ldots, b_{n}\right)=\left(a_{1}+b_{1}, \ldots, a_{n}+b_{n}\right)+Z+\tau(Z),
$$

where $Z$ is a sum of a-first terms and $\tau$ flips the occurrence of $a_{i}$ and $b_{i}$ for all $i$. In particular, the product is commutative.

\footnotetext{
${ }^{1}$ When calculated symbolically.
} 


\section{Example 1}

$$
\begin{aligned}
\left(a_{1}, a_{2}\right) \cdot\left(b_{1}, b_{2}\right)= & \left(a_{1}+b_{1}, a_{2}+b_{2}\right) \\
& +\left(a_{1}+b_{1}, a_{2}, b_{2}\right)+\left(a_{1}, b_{1}, a_{2}+b_{2}\right)+\left(a_{1}, b_{1}+a_{2}, b_{2}\right) \\
& +\left(a_{1}, a_{2}, b_{1}, b_{2}\right)+\left(a_{1}, b_{1}, a_{2}, b_{2}\right)+\left(a_{1}, b_{1}, b_{2}, a_{2}\right) \\
& +\left(b_{1}+a_{1}, b_{2}, a_{2}\right)+\left(b_{1}, a_{1}, b_{2}+a_{2}\right)+\left(b_{1}, a_{1}+b_{2}, a_{2}\right) \\
& +\left(b_{1}, b_{2}, a_{1}, a_{2}\right)+\left(b_{1}, a_{1}, b_{2}, a_{2}\right)+\left(b_{1}, a_{1}, a_{2}, b_{2}\right),
\end{aligned}
$$

where the second line consists of $a$-first terms and the third line is the $\tau$-image of the second line.

Corollary 1 For $A=\left(a_{1}, \ldots, a_{n}\right)$,

$$
A \cdot A=\left(2 a_{1}, \ldots, 2 a_{n}\right), \quad A^{2^{m}}=\left(2^{m} a_{1}, \ldots, 2^{m} a_{n}\right) .
$$

Proof In this case, the flip map $\tau$ in Lemma 1 is the identity.

It is easy to see from the duality relation $\left\langle S_{I} S_{J}, S^{K}\right\rangle=\left\langle S_{I} \otimes S_{J}, \Delta\left(S^{K}\right)\right\rangle$ that the product on $\mathcal{F}_{2}^{*}$ dual to (1) is given by $S_{I} S_{J}=\sum_{K \in I \cdot J} S_{K}$.

\section{Dual Steenrod algebra as a sub-Hopf algebra of $\mathcal{F}_{2}^{*}$}

To identify the image of the inclusion $\pi^{*}: \mathcal{A}_{2}^{*} \rightarrow \mathcal{F}_{2}^{*}$, we prove some lemmas in this section. Let $\xi_{n}=S q_{2^{n-1}, 2^{n-2}, \ldots, 2^{0}}$.

Lemma 2 (cf. [2,19]) We have

$$
\begin{aligned}
\pi^{*}\left(S q_{2^{n}}\right) & =S_{2^{n}}, \\
\pi^{*}\left(\xi_{n}\right) & =S_{2^{n-1}, 2^{n-2}, \ldots, 2^{0}}
\end{aligned}
$$

Proof For the first equation, we have to show that for any non-admissible sequence $I$, the right-hand side of

$$
S q^{I}=\sum_{J: \text { admissible }} C_{J}^{I} S q^{J}
$$

does not contain $S q^{2^{n}}$. If there exists such an $I$, we can assume it has length two, that is, $I=(i, j)$. (Because the right-hand side is obtained by successively applying the length two relations.) By the Adem relations in Eq. (2), we have $i+j=2^{n}$ and

$$
1 \equiv\left(\begin{array}{c}
j-1 \\
i
\end{array}\right) \equiv\left(\begin{array}{c}
2^{n}-1-i \\
i
\end{array}\right) \bmod 2 .
$$

However, the binary expressions of $2^{n}-1-i$ and $i$ are complementary and the binary expression of $2^{n}-1-i$ contains at least one digit with 0 . Hence, by Lucas' Theorem, we have $\left(\begin{array}{c}2^{n}-1-i \\ i\end{array}\right) \equiv 0 \bmod 2$; we arrive at a contradiction. 
For the second equation, suppose that there exists an $I=(i, j)$ such that $i<2 j$ and

$$
S q^{i, j}=\sum_{k=0}^{\lfloor i / 2\rfloor}\left(\begin{array}{c}
j-k-1 \\
i-2 k
\end{array}\right) S q^{i+j-k} S q^{k}
$$

contains $S q^{2^{n-k}}$ or $S q^{2^{n-k}} S q^{2^{n-k-1}}$ as a summand. The former case is already ruled out by the first equation. For the latter case to happen, we should have

$$
i+j=2^{n-k}+2^{n-k-1}, \quad\lfloor i / 2\rfloor \geq 2^{n-k-1} .
$$

But this implies $j \leq 2^{n-k-1}$ so $i \geq 2 j$; we arrive at a contradiction.

Put $\bar{\xi}_{n}=\pi^{*}\left(\xi_{n}\right)=S_{2^{n-1}, 2^{n-2}, \ldots, 2^{0}}$. We denote by $\widetilde{\mathcal{A}}_{2}^{*}$ the subalgebra of $\mathcal{F}_{2}^{*}$ generated by $\left\{\bar{\xi}_{n} \mid 0<n\right\}$. For a sequence $L=\left(l_{1}, l_{2}, \ldots, l_{n}\right)$ of non-negative integers, we denote $\bar{\xi}_{1}^{l_{1}} \bar{\xi}_{2} l_{2} \cdots \bar{\xi}_{n}^{l_{n}}$ by $\bar{\xi}^{L}$. Then, the monomials $\bar{\xi}^{L}$ span $\widetilde{\mathcal{A}}_{2}^{*}$. Now, we identify $\widetilde{\mathcal{A}}_{2}^{*}$ with $\operatorname{Im}\left(\pi^{*}\right)$.

Recall the definition of the excess vector of an admissible sequence $J=$ $\left(j_{1}, j_{2}, \ldots, j_{n}\right)$ :

$$
\gamma\left(j_{1}, j_{2}, \ldots, j_{n}\right)=\left(j_{1}-2 j_{2}, j_{2}-2 j_{3}, \ldots, j_{n-1}-2 j_{n}, j_{n}\right)
$$

This gives a bijection between admissible sequences and sequences of non-negative integers. The inverse is given by

$$
\gamma^{-1}\left(l_{1}, l_{2}, \ldots, l_{n}\right)=\left(l_{1}+2 l_{2}+2^{2} l_{3}+\cdots+2^{n-1} l_{n}, \ldots, l_{n-1}+2 l_{n}, l_{n}\right) .
$$

We put the right lexicographic order on $\mathcal{W}$, i.e.,

$$
\left(a_{1}, a_{2}, \ldots, a_{n}\right)>\left(b_{1}, b_{2}, \ldots, b_{m}\right) \Leftrightarrow(n>m) \text { or }\left(\exists k, a_{k}>b_{k} \text { and } a_{i}=b_{i} \forall i>k\right) \text {. }
$$

This induces an ordering on the basis elements $S_{I}$ which is compatible with the overlapping shuffle product. Observe that the lowest term in the product $S_{I} \cdot S_{I^{\prime}}$ for $I=\left(i_{1}, i_{2}, \ldots\right)$ and $I^{\prime}=\left(i_{1}^{\prime}, i_{2}^{\prime}, \ldots\right)$ is $S_{\left(i_{1}+i_{1}^{\prime}, i_{2}+i_{2}^{\prime}, \ldots\right)}$.

Lemma 3 For an admissible sequence J,

$$
\left\langle\bar{\xi}^{\gamma(J)}, S^{I}\right\rangle= \begin{cases}1 & (I=J) \\ 0 & (I<J)\end{cases}
$$

Proof We proceed by induction on $J=\left(j_{1}, \ldots, j_{n}\right)$. Put $J^{\prime}=\left(j_{1}-2^{n-1}, j_{2}-\right.$ $\left.2^{n-2}, \ldots, j_{n}-2^{0}\right)$. Then by induction hypothesis,

$$
\bar{\xi}^{\gamma\left(J^{\prime}\right)}=S_{J^{\prime}}+\left(\text { terms higher than } S_{J^{\prime}}\right)
$$


It follows that

$$
\begin{aligned}
\bar{\xi}^{\gamma(J)} & =\bar{\xi}^{\gamma\left(J^{\prime}\right)} \cdot \bar{\xi}_{n} \\
& =\left(S_{J^{\prime}}+\left(\text { terms higher than } S_{J^{\prime}}\right)\right) \cdot S_{2^{n-1}, 2^{n-2}, \ldots, 2^{0}} \\
& =S_{J}+\left(\text { terms higher than } S_{J}\right) .
\end{aligned}
$$

By this upper-triangularity, the monomials $\bar{\xi}^{L}$ are linearly independent and we have

\section{Theorem 1}

$$
\operatorname{Im}\left(\pi^{*}\right)=\widetilde{\mathcal{A}}_{2}^{*}=\mathbb{F}_{2}\left[\bar{\xi}_{1}, \bar{\xi}_{2}, \ldots,\right]
$$

Proof By Lemma 3 in each degree $\widetilde{\mathcal{A}}_{2}^{*}$ has the same dimension as $\mathcal{A}_{2}^{*}$ (the number of admissible sequences).

This is nothing but the well-known fact:

Corollary 2 [13]

$$
\mathcal{A}_{2}^{*}=\mathbb{F}_{2}\left[\xi_{1}, \xi_{2}, \ldots,\right]
$$

where

$$
\xi^{\gamma(J)}=S q_{J}+\left(\text { terms higher than } S q_{J}\right)
$$

\section{Computation with $\pi^{*}$}

Recall from [19, Section 4] the linear left inverse $r: \mathcal{F}_{2}^{*} \rightarrow \mathcal{A}_{2}^{*}$ of $\pi^{*}$ :

$$
r\left(S_{I}\right)= \begin{cases}S q_{I} & (I: \text { admissible }) \\ 0 & \text { (otherwise })\end{cases}
$$

For (ii) of Problem 1, we can compute

$$
\begin{aligned}
\xi^{\left(l_{1}, l_{2}, \ldots, l_{n}\right)} & =r \pi^{*}\left(\xi^{\left(l_{1}, l_{2}, \ldots, l_{n}\right)}\right) \\
& =r\left(\bar{\xi}_{1}^{l_{1}} \bar{\xi}_{2}^{l_{2}} \ldots \bar{\xi}_{n}^{l_{n}}\right) \\
& =r\left(\left(S_{2^{0}}\right)^{l_{1}}\left(S_{2^{1}, 2^{0}}\right)^{l_{2}} \cdots\left(S_{2^{n-1}, 2^{n-2}, \ldots, 2^{0}}\right)^{l_{n}}\right)
\end{aligned}
$$

and it reduces to computing admissible sequences occurring in the overlapping shuffle product.

For (i) of Problem 1, by Corollary 2 we have

$$
\pi^{*}\left(\xi^{\gamma(J)}\right)=\pi^{*}\left(S q_{J}+\left(\text { terms higher than } S q_{J}\right)\right)
$$


and the left-hand side can be computed by the overlapping shuffle product. Thus, we can compute inductively the coefficients $C_{J}^{I}$ in

$$
\pi^{*}\left(S q_{J}\right)=\sum_{I} C_{J}^{I} S_{I}
$$

We implemented the algorithm into a Maple code [16].

Example 2 We demonstrate the above algorithm in low degrees. First, compute $\pi^{*}$ image of monomials $\xi^{L}$ :

$$
\begin{aligned}
\pi^{*}\left(\xi_{2}^{2}\right)= & S_{2,1} S_{2,1}=S_{4,2} \\
\pi^{*}\left(\xi_{1}^{3} \xi_{2}\right)= & \left(S_{3}+S_{1,2}+S_{2,1}\right) S_{2,1} \\
= & S_{5,1}+S_{4,2}+S_{3,3}+S_{2,4}+S_{2,3,1}+S_{1,4,1} \\
& +S_{3,1,2}+S_{2,2,2}+S_{1,2,3}+S_{2,1,2,1}+S_{1,2,1,2} \\
\pi^{*}\left(\xi_{1}^{6}\right)= & S_{6}+S_{4,2}+S_{2,4} .
\end{aligned}
$$

Taking $r$ on the both sides of equations, we obtain

$$
\xi_{2}^{2}=S q_{4,2}, \quad \xi_{1}^{3} \xi_{2}=S q_{5,1}+S q_{4,2}, \quad \xi_{1}^{6}=S q_{6}+S q_{4,2}
$$

Again taking $\pi^{*}$ on the both sides of the equations, we obtain

$$
\begin{aligned}
\pi^{*}\left(S q_{4,2}\right)= & S_{4,2} \\
\pi^{*}\left(S q_{5,1}+S q_{4,2}\right)= & S_{5,1}+S_{4,2}+S_{3,3}+S_{2,4}+S_{2,3,1}+S_{1,4,1} \\
& +S_{3,1,2}+S_{2,2,2}+S_{1,2,3}+S_{2,1,2,1}+S_{1,2,1,2} \\
\pi^{*}\left(S q_{6}+S q_{4,2}\right)= & S_{6}+S_{4,2}+S_{2,4} .
\end{aligned}
$$

Finally, by using the upper-triangularity, we obtain

$$
\begin{aligned}
\pi^{*}\left(S q_{4,2}\right)= & S_{4,2} \\
\pi^{*}\left(S q_{5,1}\right)= & S_{5,1}+S_{3,3}+S_{2,4}+S_{2,3,1}+S_{1,4,1}+S_{3,1,2}+S_{2,2,2} \\
& +S_{1,2,3}+S_{2,1,2,1}+S_{1,2,1,2} \\
\pi^{*}\left(S q_{6}\right)= & S_{6}+S_{2,4} .
\end{aligned}
$$

\section{Formula for the conjugation}

Any connected commutative or co-commutative Hopf algebra has a unique conjugation $\chi$ satisfying

$$
\chi(1)=1, \quad \chi(x y)=\chi(y) \chi(x), \quad \chi^{2}(x)=x, \quad \sum x^{\prime} \chi\left(x^{\prime \prime}\right)=0,
$$


where $\Delta(x)=\sum x^{\prime} \otimes x^{\prime \prime}$ and $\operatorname{deg}(x)>0$ [14]. The conjugation invariants in $\mathcal{A}_{2}^{*}$ is studied in [5] because it is relevant to the commutativity of ring spectra [1, Lecture 3]. The same problem in $\mathcal{F}_{2}^{*}$ has been also studied in $[3,4]$. Here we investigate them through our point of view.

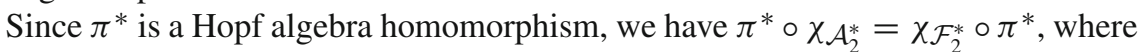
$\chi_{\mathcal{A}_{2}^{*}}$ and $\chi_{\mathcal{F}_{2}^{*}}$ denote the conjugation operations in $\mathcal{A}_{2}^{*}$ and $\mathcal{F}_{2}^{*}$ respectively. For the module basis $S_{I}$ in $\mathcal{F}_{2}^{*}$, the conjugation $\chi_{\mathcal{F}_{2}^{*}}$ is calculated combinatorially.

Definition 2 The coarsening set $C(I)$ of a sequence $I=\left(i_{1}, \ldots, i_{l}\right)$ is defined recursively as

$$
C(I):=\left\{\left(i_{1}, I^{\prime}\right),\left(i_{1}+i_{1}^{\prime}, I_{2}^{\prime}\right) \mid I^{\prime} \in C\left(\left(i_{2}, \ldots, i_{l}\right)\right)\right\} \quad \text { and } \quad C((i))=\{(i)\}
$$

where $I_{2}^{\prime}$ is the tail partial sequence $\left(i_{2}^{\prime}, \ldots, i_{l^{\prime}}^{\prime}\right)$ of $I^{\prime}=\left(i_{1}^{\prime}, i_{2}^{\prime}, \ldots, i_{l^{\prime}}^{\prime}\right)$.

Example $3 C((a, b, c))=\{(a, b, c),(a+b, c),(a, b+c),(a+b+c)\}$.

A formula for the conjugation operation in the dual Leibniz-Hopf algebra is given by Ehrenborg [6, Proposition 3.4]. We now give a simple proof for its mod 2 reduction.

\section{Proposition 1}

$$
\chi \mathcal{F}_{2}^{*}\left(S_{I}\right)=\sum_{I^{\prime} \in C\left(I^{-1}\right)} S_{I^{\prime}}
$$

where $I^{-1}=\left(i_{l}, \ldots, i_{1}\right)$ is the reverse sequence of $I=\left(i_{1}, \ldots, i_{l}\right)$.

Proof The conjugation is uniquely characterised by

$$
\chi_{\mathcal{F}_{2}^{*}}(1)=1, \quad \sum x^{\prime} \chi_{\mathcal{F}_{2}^{*}}\left(x^{\prime \prime}\right)=0
$$

where $\Delta(x)=\sum x^{\prime} \otimes x^{\prime \prime}$ and $\operatorname{deg}(x)>0$. We put $\chi^{\prime}\left(S_{I}\right)=\sum_{I^{\prime} \in C\left(I^{-1}\right)} S_{I^{\prime}}$ and show that it satisfies the above equations. It is obvious that $\chi^{\prime}(1)=1$. Since the co-product is given in (3), the second equation reads

$$
\sum_{k=0}^{l} S_{i_{1}, \ldots, i_{k}} \chi^{\prime}\left(S_{i_{k+1}, \ldots, i_{n}}\right)=0 \quad\left(\forall I=\left(i_{1}, i_{2}, \ldots, i_{n}\right)\right) .
$$

We regard an element of $\mathbb{F}_{2}\langle\mathcal{W}\rangle$ with a finite subset of $\mathcal{W}$ in the obvious way. We investigate relation between coarsening and the overlapping shuffle product. Define

$$
C_{k}(I)=\sum_{I^{\prime} \in C\left(\left(I_{k+1}\right)^{-1}\right)} I^{\prime} \cdot\left(i_{1}, \ldots, i_{k}\right)
$$


We observe ${ }^{2}$ that $C\left(I^{-1}\right) \subset C_{1}(I)$ and $C_{1}^{\prime}(I):=C_{1}(I) \backslash C\left(I^{-1}\right)$ consists of those sequences that $i_{1}$ appears to the left of $i_{2}$. In turn, $C_{1}^{\prime}(I) \subset C_{2}(I)$ and $C_{2}^{\prime}(I):=$ $C_{2}(I) \backslash C_{1}^{\prime}(I)$ consists of those sequences that $i_{2}$ appears to the left of $i_{3}$. Continuing similarly, we obtain

$$
C\left(I^{-1}\right)=\sum_{k=1}^{l} C_{k}(I)
$$

It follows that

$$
\chi^{\prime}\left(S_{I}\right)=\sum_{k=1}^{l} \sum_{I^{\prime} \in C\left(\left(I_{k+1}\right)^{-1}\right)} S_{\left(i_{1}, \ldots, i_{k}\right)} \cdot S_{I^{\prime}}=\sum_{k=0}^{l} S_{i_{1}, \ldots, i_{k}} \chi^{\prime}\left(S_{i_{k+1}, \ldots, i_{n}}\right)-\chi^{\prime}\left(S_{I}\right)
$$

and $\sum_{k=0}^{l} S_{i_{1}, \ldots, i_{k}} \chi^{\prime}\left(S_{i_{k+1}, \ldots, i_{n}}\right)=0$.

We give another formula for $\chi_{\mathcal{F}_{2}^{*}}\left(S_{I}\right)$.

Definition 3 For a sequence $a_{1}, a_{2}, \ldots, a_{n}$, the set of ordered block partitions $\mathcal{P}\left(a_{1}, a_{2}, \ldots, a_{n}\right)$ consists of elements of the form

$$
\beta=\left(\left(a_{1}, a_{2}, \ldots, a_{i_{1}}\right)\left|\left(a_{i_{1}+1}, \ldots, a_{i_{2}}\right)\right| \ldots \mid\left(a_{i_{l-1}+1}, \ldots, a_{i_{l}}\right)\right),
$$

where $1 \leq i_{1}<i_{2}<\cdots<i_{l}=n$. We denote $l(\beta)=l$ and $\beta(k)=\left(a_{i_{k-1}+1}, \ldots, a_{i_{k}}\right)$. Or inductively, we can define

$$
\mathcal{P}\left(a_{1}, a_{2}, \ldots, a_{n}\right)=\bigcup_{k=1}^{n}\left\{\left(\left(a_{1}, \ldots, a_{k}\right) \mid \beta\right) \mid \beta \in \mathcal{P}\left(a_{k+1}, a_{k+2}, \ldots, a_{n}\right)\right\} .
$$

Theorem 2

$$
\chi_{\mathcal{F}_{2}^{*}}\left(S_{I}\right)=\sum_{\beta \in \mathcal{P}(I)} \prod_{k=1}^{l(\beta)} S_{\beta(k)}
$$

Proof Let $I=\left(a_{1}, a_{2}, \ldots, a_{n}\right)$. Put

$$
\chi^{\prime}\left(S_{a_{1}, a_{2}, \ldots, a_{n}}\right)=\sum_{\beta \in \mathcal{P}\left(a_{1}, a_{2}, \ldots, a_{n}\right)} \prod_{k=1}^{l(\beta)} S_{\beta(k)}
$$

\footnotetext{
2 Here, we deal with sequences symbolically so that we avoid cancellations like $\left(i_{3}+i_{2}, i_{1}\right)+\left(i_{3}+i_{1}, i_{2}\right)=$ 0 when $i_{1}=i_{2}$.
} 
and we check that

$$
\chi^{\prime}(1)=1, \quad \sum_{k=0}^{n} S_{a_{1}, \ldots, a_{k}} \chi^{\prime}\left(S_{a_{k+1}, \ldots, a_{n}}\right)=0 .
$$

Then, by the uniqueness of the conjugation, we have $\chi_{\mathcal{F}_{2}^{*}}=\chi^{\prime}$. The first assertion is trivial. For the second, observe that by (7)

$$
\begin{aligned}
\chi^{\prime}\left(S_{a_{1}, a_{2}, \ldots, a_{n}}\right) & =\sum_{k=1}^{n} S_{a_{1}, \ldots, a_{k}}\left(\sum_{\beta \in \mathcal{P}\left(a_{k+1}, a_{k+2}, \ldots, a_{n}\right)} \prod_{j=1}^{l(\beta)} S_{\beta(j)}\right) \\
& =\sum_{k=1}^{n} S_{a_{1}, \ldots, a_{k}} \chi^{\prime}\left(S_{a_{k+1}, \ldots, a_{n}}\right) .
\end{aligned}
$$

Hence, we have

$$
\begin{aligned}
\sum_{k=0}^{n} S_{a_{1}, \ldots, a_{k}} \chi^{\prime}\left(S_{a_{k+1}, \ldots, a_{n}}\right) & =\chi^{\prime}\left(S_{a_{1}, a_{2}, \ldots, a_{n}}\right)+\sum_{k=1}^{n} S_{a_{1}, \ldots, a_{k}} \chi^{\prime}\left(S_{a_{k+1}, \ldots, a_{n}}\right) \\
& =2 \chi^{\prime}\left(S_{a_{1}, a_{2}, \ldots, a_{n}}\right) \\
& =0 .
\end{aligned}
$$

\section{Example 4}

$$
\begin{aligned}
\chi_{\mathcal{F}_{2}^{*}}\left(S_{1,2,3}\right) & =S_{3,2,1}+S_{5,1}+S_{3,3}+S_{6} \\
& =S_{1,2,3}+S_{1} S_{2,3}+S_{1,2} S_{3}+S_{1} S_{2} S_{3} .
\end{aligned}
$$

The first line is computed by Proposition 1, and the second by Theorem 2 .

Theorem 2 can be thought of as a generalisation of Milnor's conjugation formula in $\mathcal{A}_{2}^{*}$. To see this, we first show a small lemma:

\section{Lemma 4}

$$
\bar{\xi}_{n}^{2^{m}}=\left(S_{2^{n-1}, 2^{n-2}, \ldots, 2^{0}}\right)^{2^{m}}=S_{2^{n+m-1}, 2^{n+m-2}, \ldots, 2^{m}}
$$

Proof This is a direct consequence of Corollary 1 combined with Lemma 2.

Corollary 3 [13, Lemma 10]

$$
\chi_{\mathcal{A}_{2}^{*}}\left(\xi_{n}\right)=\sum_{\alpha} \prod_{k=1}^{l(\alpha)} \xi_{\alpha(k)}^{2^{\sigma(k)}},
$$

where $\alpha=(\alpha(1)|\alpha(2)| \ldots \mid \alpha(l(\alpha))$ runs through all the compositions of the integer $n$ and $\sigma(k)=\sum_{j=1}^{k-1} \alpha(j)$. 
Proof We apply the injection $\pi^{*}$ to the both sides of (8) and show that they coincide.

For the left-hand side, by Lemma 4 we have

$$
\pi^{*}\left(\chi \mathcal{A}_{2}^{*}\left(\xi_{n}\right)\right)=\chi \mathcal{F}_{2}^{*}\left(\pi^{*}\left(\xi_{n}\right)\right)=\chi \mathcal{F}_{2}^{*}\left(S_{2^{n-1}, 2^{n-2}, \ldots, 2^{0}}\right)
$$

Since $\pi^{*}\left(\xi_{\alpha(k)}^{2^{\sigma(k)}}\right)=S_{2^{\alpha(k)+\sigma(k)-1}, \ldots, 2^{\sigma(k)}}$ by Lemma 4 , we see

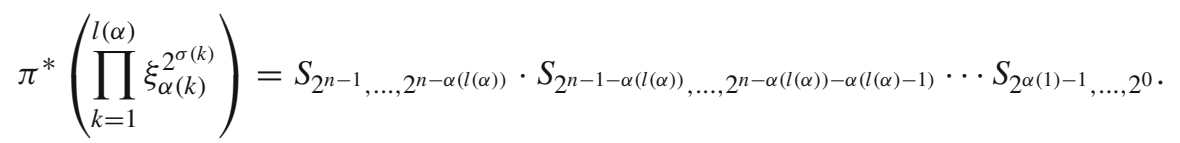

So when $\alpha$ ranges over all compositions of $n$, we get all the ordered block partitions of the sequence $2^{n-1}, 2^{n-2}, \ldots, 2^{0}$. The assertion follows from Theorem 2 .

\section{Duality between $\mathcal{F}_{2}$ and $\mathcal{F}_{2}^{*}$}

In the previous section we discussed how to compute the conjugation in $\mathcal{F}_{2}^{*}$. Here, we relate the conjugation in $\mathcal{F}_{2}$ with that in $\mathcal{F}_{2}^{*}$ by using a self-duality of $\mathcal{W}$. Denote $I \preceq I^{\prime}$ if $I \in C\left(I^{\prime}\right)$. We think of $I \in \mathcal{W}$ as a string of 1 's separated by ' + ' and commas; $(\underbrace{1+1+\cdots+1}_{i_{1}}, \underbrace{1+1+\cdots+1}_{i_{2}}, \ldots, \underbrace{1+1+\cdots+1}_{i_{l}})$.

Definition 4 We define the dual $\bar{I} \in \mathcal{W}$ of $I$ by switching + and the commas.

Example 5 For $I=(1,3,2)=(1,1+1+1,1+1)$, its dual is

$$
\bar{I}=(1+1,1,1+1,1)=(2,1,2,1) .
$$

It is easily seen that $\overline{\bar{I}}=I$ and $I \preceq I^{\prime} \Leftrightarrow \bar{I} \succeq \bar{I}^{\prime}$. Extend the duality to one between $\mathcal{F}_{2}$ and $\mathcal{F}_{2}^{*}$ by

$$
D\left(S^{I}\right)=S_{\bar{I}}, \quad D^{-1}\left(S_{I}\right)=S^{\bar{I}}
$$

Theorem 3 We have $D \circ \chi_{\mathcal{F}_{2}}=\chi_{\mathcal{F}_{2}^{*}} \circ D$. In particular, $f \in \mathcal{F}_{2}$ is a conjugation invariant if and only if so is $\bar{f} \in \mathcal{F}_{2}^{*}$.

Proof We compute

$$
\begin{aligned}
D^{-1} \circ \chi_{\mathcal{F}_{2}^{*}} \circ D\left(S^{I}\right) & =D^{-1} \chi_{\mathcal{F}_{2}^{*}}\left(S_{\bar{I}}\right)=D^{-1}\left(\sum_{I^{\prime} \preceq(\bar{I})^{-1}} S_{I^{\prime}}\right)=D^{-1}\left(\sum_{\bar{I}^{\prime} \succeq I^{-1}} S_{I^{\prime}}\right) \\
& =\sum_{\bar{I}^{\prime} \succeq I^{-1}} S^{\bar{I}^{\prime}}=\sum_{I^{\prime} \succeq I^{-1}} S^{I^{\prime}} .
\end{aligned}
$$


Put $\chi^{\prime}\left(S^{I}\right)=\sum_{I^{\prime} \succeq I^{-1}} S^{I^{\prime}}$. Then, one can check $\chi^{\prime}(1)=1$ and $\sum x^{\prime} \chi^{\prime}\left(x^{\prime \prime}\right)=0$ for $\Delta x=\sum x^{\prime} \otimes x^{\prime \prime}$ as in Proposition 1. Hence, by the uniqueness of the conjugation, we have $\chi^{\prime}=\chi \mathcal{F}_{2}$.

Example $6 f=S^{1,1,2}+S^{2,1,1}+S^{1,1,1,1}$ is a $\chi \mathcal{F}_{2}$-invariant, whilst $D(f)=S_{3,1}+$ $S_{1,3}+S_{4}$ is a $\chi_{\mathcal{F}_{2}^{*} \text {-invariant. }}$

Remark 1 The sub-module of the conjugation invariants in $\mathcal{F}_{2}$ is $\operatorname{ker}\left(\chi_{\mathcal{F}_{2}}-1\right)$ and that in $\mathcal{F}_{2}^{*}$ is $\operatorname{ker}\left(\chi_{\mathcal{F}_{2}^{*}}-1\right)$. The conjugations in $\mathcal{F}_{2}$ and $\mathcal{F}_{2}^{*}$ are dual to each other, and hence, the linear map $\chi \mathcal{F}_{2}-1$ is transpose to $\chi \mathcal{F}_{2}^{*}-1$ with the kernel of same dimension [3]. Theorem 3 gives more information by specifying an explicit correspondence between their elements.

Acknowledgements We would like to thank Stephen Theriault, Martin Crossley, and Carmen Rovi for their comments on the earlier version of this paper.

\section{References}

1. Adams, J.F.: Lectures on generalised cohomology. Springer Lect. Notes Math. 99, 1-138 (1969)

2. Crossley, M.D.: Some Hopf algebras of words. Glasgow Math J. 48, 575-582 (2006)

3. Crossley, M.D., Turgay, N.D.: Conjugation invariants in the Leibniz Hopf algebra. J. Pure Appl. Algebra 217, 2247-2254 (2013)

4. Crossley, M.D., Turgay, N.D.: Conjugation invariants in the mod 2 dual Leibniz-Hopf algebra. Commun. Algebra 41, 3261-3266 (2013)

5. Crossley, M.D., Whitehouse, S.: On conjugation invariants in the dual Steenrod algebra. Proc. AMS. 128, 2809-2818 (2000)

6. Ehrenborg, R.: On posets and Hopf algebras. Adv. Math. 119, 1-25 (1996)

7. Gelfand, I.M., Krob, D., Lascoux, A., Leclerc, B., Retakh, V.S., Thibon, J.-Y.: Noncommutative symmetric functions. Adv. Math. 112, 218-348 (1995)

8. Hazewinkel, M.: Generalized overlapping shuffle algebras. J. Math. Sci. N Y 106, 3168-3186 (2001)

9. Hazewinkel, M.: The algebra of quasi-symmetric functions is free over the integers. Adv. Math. 164, 283-300 (2001)

10. Hazewinkel, M.: Symmetric functions, noncommutative symmetric functions, and quasisymmetric functions. Monodromy and differential equations. Acta Appl. Math. 75, 55-83 (2003)

11. Hazewinkel, M.: Explicit polynomial generators for the ring of quasisymmetric functions over the integers. Acta Appl. Math 109, 39-44 (2010)

12. Malvenuto, C., Reutenauer, C.: Duality between quasi-symmetric functions and the solomon descent algebra. J. Algebra 177, 967-982 (1995)

13. Milnor, J.: The Steenrod algebra and its dual. Ann. Math. 67, 150-171 (1958)

14. Milnor, J., Moore, J.: On the structure of Hopf algebras. Ann. Math. 81, 211-264 (1965)

15. Monks, K.G.: Change of basis, monomial relations, and the $P_{t}^{s}$ bases for the Steenrod algebra. J. Pure Appl. Algebra 125, 235-260 (1998)

16. Kaji, S.: Maple script for conjugation and product in the (dual) Leibniz-Hopf algebra and Steenrod algebra. http://www.skaji.org/code

17. Solomon, L.: A Mackey formula in the group ring of a Coxeter group. J. Algebra 41, 255-268 (1976)

18. Steenrod, N.E., Epstein, D.B.A.: Cohomology Operations. Princeton Univ. Press, Princeton (1962)

19. Turgay, N.D.: An alternative approach to the Adem relations in the mod 2 Steenrod algebra. Turkish J. Math. 38(5), 924-934 (2014) 